\title{
Interactive workshop to develop implementation framework (i-PARIHS) resources to support practice facilitation
}

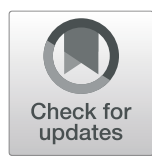

\author{
Sarah C. Hunter ${ }^{1,2^{*}}$ (D) Bo Kim ${ }^{3,4}$ and Alison L. Kitson ${ }^{1,2}$
}

\begin{abstract}
Background: Practice facilitation is a method used to address the complexity associated with implementation of innovations into primary care. To provide support, we propose that the i-PARIHS (Integrated Promoting Action on Research Implementation in Health Services) framework could support practice facilitators. The i-PARIHS framework positions facilitation as a core element for successful implementation. Therefore, the aim of this study was to provide support to practice facilitators whilst simultaneously gaining feedback on what facilitators in practice need in regard to support with operationalising the i-PARIHS framework in practice.
\end{abstract}

Methods: This study involved the delivery of a 1-h workshop on the i-PARIHS framework at the 2018 International Conference on Practice Facilitation. The authors provided an overview of the i-PARIHS framework, how it can be used to support the facilitation of innovations into practice, and finally, attendees worked through facilitation scenarios and applied an i-PARIHS resource. At the end of the workshop, attendees were invited to participant in the research component, by completing a post-workshop survey on the workshop content and the i-PARIHS resource.

Results: Participants were highly engaged and enthusiastic about the workshop. Participants reported that an introduction to implementation frameworks was valuable and the example of how the i-PARIHS framework had been used in a previous project was helpful. Overall, this study identifies how framework informed facilitation helped participants feel more equipped to conduct systematic facilitation and that the development of i-PARIHS resources would be helpful in their everyday work.

Conclusions: This study demonstrates that the existence of implementation frameworks is not sufficient to provide support to those who facilitate in the real world. The current study introduced practice facilitators to the i-PARIHS framework, and the findings demonstrate the need to develop and refine existing i-PARIHS resources to support facilitation. Specifically, the next steps stemming from this study will be to (i) continue to utilize workshops for sharing and refining tools, (ii) allocate development efforts to tools that assist with planning, (iii) focus on tool provision mechanisms that keep user-friendliness in mind, and (iv) translate the i-PARIHS facilitation checklist from academic language into more practical and user-friendly language.

Keywords: Practice facilitation, Implementation science, Knowledge translation, i-PARIHS framework

\footnotetext{
* Correspondence: sarah.hunter@flinders.edu.au

'College of Nursing and Health Sciences, Flinders University, Sturt Road,

Bedford Park, Adelaide, South Australia 5042, Australia

${ }^{2}$ Caring Futures Institute, Flinders University, Sturt Road, Bedford Park,

Adelaide, South Australia 5042, Australia

Full list of author information is available at the end of the article
}

(c) The Author(s). 2020 Open Access This article is licensed under a Creative Commons Attribution 4.0 International License, which permits use, sharing, adaptation, distribution and reproduction in any medium or format, as long as you give appropriate credit to the original author(s) and the source, provide a link to the Creative Commons licence, and indicate if changes were made. The images or other third party material in this article are included in the article's Creative Commons licence, unless indicated otherwise in a credit line to the material. If material is not included in the article's Creative Commons licence and your intended use is not permitted by statutory regulation or exceeds the permitted use, you will need to obtain permission directly from the copyright holder. To view a copy of this licence, visit http://creativecommons.org/licenses/by/4.0/ The Creative Commons Public Domain Dedication waiver (http://creativecommons.org/publicdomain/zero/1.0/) applies to the data made available in this article, unless otherwise stated in a credit line to the data. 


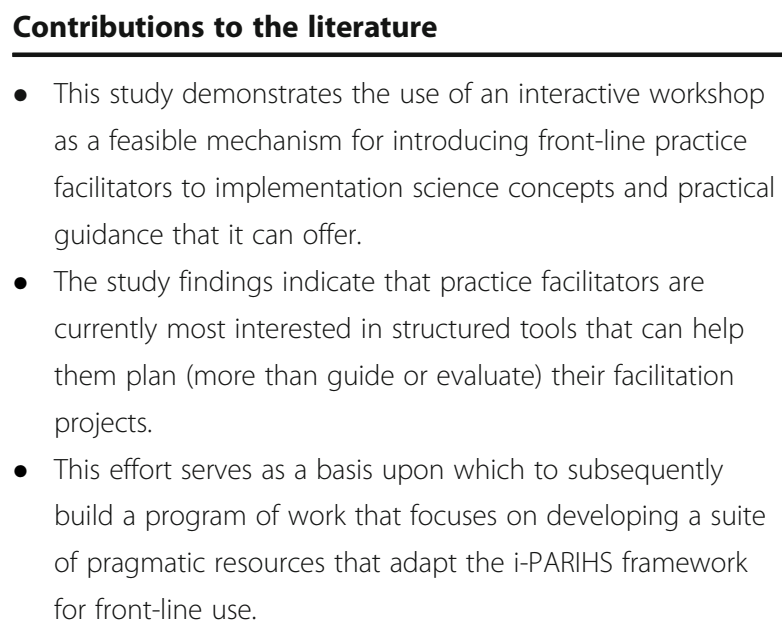

- This study demonstrates the use of an interactive workshop as a feasible mechanism for introducing front-line practice facilitators to implementation science concepts and practical guidance that it can offer.

- The study findings indicate that practice facilitators are currently most interested in structured tools that can help them plan (more than guide or evaluate) their facilitation projects.

- This effort serves as a basis upon which to subsequently build a program of work that focuses on developing a suite of pragmatic resources that adapt the i-PARIHS framework for front-line use.

\section{Background}

Successful implementation of new innovations and evidence into clinical practice can be very challenging and time-consuming [1]. Practice facilitation is a commonly used and practical method for supporting implementation in the context of primary care. Practice facilitation is a multifaceted approach involving skilled individuals who enable others through a multitude of activities (e.g. stakeholder engagement, provider feedback, and education) to address challenges associated with implementation [2].

Practice facilitation is underpinned by the understanding that primary care practices are frequently underresourced, often lack the implementation experience and skills required, and of diverse contexts [3]. This complexity of primary care is what makes practice facilitation a successful implementation strategy, engaging a dedicated 'facilitator' (a role) to enable 'facilitation' (a process) of evidence-based innovations into practice. Facilitation, as an implementation strategy, can be defined as a process of enabling groups or teams to work together effectively to achieve a common goal [4]. Facilitation is a method that focuses not just on behaviour change, but also works toward changing the way individuals think and team culture $[5,6]$. A systematic review identified that when facilitation is enacted in a way that tailors the implementation to the context and needs of the primary care setting, have significantly larger effect sizes [2]. Despite the success associated with practice facilitation, the level of complexity and tailoring involved can prove challenging for the facilitators. Practice facilitators are required to be mindful of each factor associated with facilitating innovations into practice not only during facilitation, but also must keep track of its status throughout facilitation (i.e. planning, guiding, and evaluation).

The profession of practice facilitation has grown substantially over the past decade with support from the Agency for Healthcare Research and Quality (AHRQ), emergence of multiple practice facilitator training programs, and an increasing interest in the research around knowledge translation [7]. Implementation science is an academic field that focuses on this research by developing and refining various approaches and theories to inform systematic implementation of innovations into healthcare systems [8]. These implementation theories, frameworks, and models can support practice facilitation by guiding how to predict, plan, and evaluate implementation efforts.

One such framework is the integrated-Promoting Action on Research Implementation in Health Services (iPARIHS) framework [5], which highlights facilitation as the active ingredient for implementing innovations into practice. The i-PARIHS framework is the revised iteration of the original PARIHS framework and responds to several criticisms relating to the need for practical tools and case studies to help clinicians and researchers operationalise the framework $[5,6]$. The i-PARIHS framework specifies core constructs and sub-constructs, which influence successful implementation, and aligns with practice facilitation, by being one of the first frameworks with an underlying philosophy that implementing research into healthcare practice is complex, unpredictable, and non-linear $[5,6]$.

Unfortunately, the existence of frameworks alone does not provide explicit guidance for facilitators. Like many frameworks, the original PARIHS framework received criticism for being difficult to operationalise, whereby resources and support are needed in order to effectively operationalise the constructs and how they relate to one another [9]. Therefore, in addition to the revised theory and clearly specified framework constructs, the developers of i-PARIHS provided several tools to operationalize i-PARIHS in practice, outlined in their published facilitation guide $[5,6]$. This includes the $\mathrm{Fa}$ cilitation Checklist to support structured assessment of the framework constructs. See Table 1 for some examples of the questions from the Facilitation Checklist.

The Facilitation Checklist provides facilitators with various reflective questions to consider when working through and assessing the other i-PARIHS constructs (the innovation, recipients, and context). However, whilst these questions provide more operational support than the original PARIHS framework, it is important to evaluate whether the i-PARIHS Facilitation Checklist provides users with the appropriate support they need in order to successfully undertake facilitation.

Therefore, the current study took the i-PARIHS Facilitation Checklist to the North American Primary Care Research Group (NAPCRG)'s 2018 International Conference on Practice Facilitation, Tampa, Florida. This conference brings together a large group of facilitators, researchers, and clinicians who are interested in practice facilitation. The focus of the 2018 conference was on 
Table 1 Example of i-PARIHS Facilitation Checklist questions

i-PARIHS Facilitation Checklist questions
Characteristics of the innovation
Who is likely to be affected by the proposed innovation?
or change?
Is the evidence packaged in an accessible and useable form
(e.g. a clinical guideline, care pathway, or algorithm)?
How much novelty does the evidence introduce?
Does it offer advantages over the current way of doing things
Is there potential to test out/pilot the introduction of the
evidence/innovation on a small scale in the first instance?
The recipients of the evidence/innovation
Do individual members of the team want to apply the change?
Do they perceive the proposed change as valuable and worthwhile?

'Building Capacity for Practice Facilitation'. Therefore, we felt this an excellent opportunity to conduct a workshop to build capacity for the attendees, whilst also evaluating the workshop in order to gain feedback around the i-PARIHS Facilitation Checklist as a resource, from those who work as facilitators 'on the ground'.

This study is part of a larger body of work that seeks to adapt the i-PARIHS framework into a suite of practical and pragmatic resources (called the Mi-PARIHS Project-Mobilising Implementation of i-PARIHS). The current small-scale study explores how helpful and useful an adapted version of the i-PARIHS Facilitation Checklist is to practice facilitators in planning, guiding, and evaluating their implementation and facilitation endeavours. We hope to use this feedback to refine the tools and continuously engage with participants throughout tool development.

Specifically, our objectives are as follows:

1. How helpful do practice facilitators find the workshop format and its components, as a way of being introduced to an i-PARIHS tool and how it can be used?

2. Do practice facilitators find an i-PARIHS tool useful and if so, in what ways do they feel it could be most useful?

3. What characteristics do practice facilitators find most useful in an i-PARIHS tool?

\section{Methods}

\section{Setting}

We conducted a 1-h workshop at the North American Primary Care Research Group (NAPCRG)'s 2018 International Conference on Practice Facilitation, Tampa, Florida. All of the conference attendees were practice facilitators or work within primary care/practice facilitation in some way. Therefore, it was an excellent opportunity to provide education and support to practice facilitators on implementation informed facilitation, whilst also providing the chance for us to gain feedback on what facilitators in practice need in regard to support with operationalising the i-PARIHS framework in. This study was approved by the Flinders University Social and Behavioural Research Ethics Committee (project number: 8223), and all participants provided informed consent.

\section{Workshop overview}

Author ALK provided a plenary 1-h presentation on facilitation within the i-PARIHS framework. Following this, authors $\mathrm{SCH}$ and BK conducted a 1-h workshop focused on the i-PARIHS framework titled 'Mi-PARIHS: Practical tools to make framework-informed implementation and facilitation easier'. This workshop presented on the background of implementation science and the i-PARIHS framework and provided an overview of a case example of how facilitators on an implementation team previously used i-PARIHS in a project (see [10] for detail on this project). The workshop then introduced attendees to the i-PARIHS Facilitation Checklist $[5,6]$. In order for attendees to try using the Facilitation Checklist, a hypothetical facilitation scenario was provided which they could work through in small groups (3-5 people). However, participants were also free to work through the Facilitation Checklist whilst considering a project they were currently working on. To make the Facilitation Checklist useable in the context of a workshop, it was adapted from questions for reflection, to an itemised instrument (see Additional file 1). This allowed attendees to respond to each question with a rating between -2 and +2 . Negative responses $(-2$ and -1$)$ indicated that the particular question was considered a barrier, a neutral response (0) indicated that the particular question was neutral, and positive responses $(+1$ and +2$)$ indicated that the particular question was considered an enabler. This rating scale was developed purely for the purpose of making the Facilitation Checklist interactive for participants and allow them to get a sense of how the questions are used to consider the constructs of the i-PARIHS framework and how they can inform facilitation strategies (through identifying enablers and barriers). To finalise the workshop, the authors' pre-populated responses were provided as an example so we could demonstrate how using a rating scale to answer the Facilitation Checklist questions enables the generation of an associated visual representation (radar diagram; see Additional file 2) to assist with identifying the constructs that require more dedicated facilitation. 


\section{Data collection}

Following the workshop, all attendees were invited to participate in the research component by completing a paper-based survey to provide their feedback on using the i-PARIHS tool (see Additional file 3 for the survey). The survey included 17 questions which included basic demographics, quantitative questions relating to their experience of the workshop and feedback on the tool, and finally three open-ended questions to provide participants an opportunity to provide more detailed feedback. Participants were provided with an information sheet, and completion of the survey was indicative of consent to participate. Those who did not want to participate in the study were informed to not complete a survey.

\section{Data analysis}

Workshop attendance was voluntary, so the number of participants that we could recruit for the evaluation survey was outside of our control. This resulted in a small sample size, and therefore, survey responses were analysed using descriptive statistics. The open-ended items were analysed qualitatively-authors $\mathrm{SCH}$ and $\mathrm{BK}$ independently reviewed the responses, conducted separate content analyses [11], and developed inductive codes for categories grounded in the data. The two authors $(\mathrm{SCH}$ and $\mathrm{BK}$ ) then met to discuss and resolve discrepancies.

\section{Results}

The following results are presented in four sections: (i) demographics of the participants, (ii) feedback on the conference workshop, (iii) feedback on the use of the tool, and (iv) feedback on the characteristics of the tool.

\section{Demographics}

As attendance to the workshop was separate to the research component, the number of workshop attendees was not recorded. On estimate, out of approximately 30 workshop attendees, 10 participants completed the evaluation survey. Of these 10 participants, most identified as working within a facilitator role (80\%) (Table 2).

The average years worked in their current role was 6 years (minimum 1 year, maximum 15 years). Harvey et al. [6] outlined a facilitation pathway from novice to experienced and expert facilitator, assuming different roles in the process of implementation. Table 2 outlines where the participants identified on this pathway.

\section{Workshop}

The response to the overall experience of the conference workshop was positive with no negative responses (Table 3).

As an introduction to the importance of implementation frameworks and information on the i-PARIHS framework, $40 \%$ of participants reported this as very
Table 2 Summary of facilitation roles and experience

\begin{tabular}{lll}
\hline Survey question & Number & Percentage \\
\hline Current professional role & 4 & 40 \\
Full-time practice facilitator & 4 & 40 \\
Part-time practice facilitator & 2 & 20 \\
Not currently a practice facilitator & 2 & 100 \\
Total & 10 & \\
Facilitation experience** & 1 & 10 \\
Expert facilitator & 6 & 60 \\
Experienced facilitator & 2 & 20 \\
Beginner or novice facilitator & 1 & 10 \\
N/A & 10 & 100 \\
Total & 10 & \\
\hline
\end{tabular}

*The other aspects of their role varied from research coordinator (1), trainer and investigator (1), manager (1), and support consultant (1)

**Harvey and Kitson $([5,6]$, pp. $80-81$ ) outline three facilitator roles based on experience

valuable, $50 \%$ as valuable, and $10 \%$ as neither valuable nor not valuable.

The case example that provided an overview of how iPARIHS can be used in a real project was largely found to be helpful, but responses to how helpful working through a hypothetical facilitation scenario with the iPARIHS tool were more mixed (Table 4).

\section{Qualitative responses}

Qualitative responses help contextualize some of the quantitative responses. Participants mentioned there was not sufficient time during the workshop to fully comprehend the case study and work through the tool. Overall though, participants found the workshop important and useful, reporting that they learnt a lot about the iPARIHS framework and the types of questions they should be asking. Further, they felt more equipped to be systematic in evaluating facilitation and how to use a tool in action. Some participants reported on the interesting nature of this work and encouraged the development of these tools that they find would be helpful in their everyday work.

\section{Use of the tool}

Overall, participants responded positively to the idea of using the tool in their everyday role. Specifically, $50 \%$ of participants stated that the tool is likely something they

Table 3 Summary of overall experience of workshop

\begin{tabular}{lll}
\hline Overall experience & Number & Percentage \\
\hline Very good & 3 & 30 \\
Good & 4 & 40 \\
Neither good nor bad & 3 & 30 \\
Total & 10 & 100 \\
\hline
\end{tabular}


Table 4 Summary of how helpful participants found the case example and the facilitation scenario

\begin{tabular}{llllll}
\hline Responses & \multicolumn{2}{c}{ Case example } & & \multicolumn{2}{c}{ Facilitation scenario } \\
\cline { 2 - 3 } \cline { 6 - 6 } \cline { 5 - 6 } & $N$ & $\%$ & & $N$ & $\%$ \\
\hline Very helpful & 3 & 30 & & 3 & 30 \\
Helpful & 4 & 40 & & 3 & 30 \\
Neither helpful or unhelpful & 2 & 20 & & 1 \\
Unhelpful & 1 & 10 & & 2 \\
Very unhelpful & 0 & 0 & & 1 & 10 \\
Total & 10 & 100 & & 10 & 100 \\
\hline
\end{tabular}

would use, $20 \%$ said they would use it, $20 \%$ said not likely, and $10 \%$ said this question was not applicable. Table 5 outlines their responses regarding specifically using the tool for planning, monitoring, or evaluating their facilitation work.

\section{Characteristics of the tool}

A unique aspect of the tool is its ability to generate a visual representation (radar diagram) of the three dimensions of i-PARIHS (innovation, recipient, context) based on the questions. Table 6 demonstrates how helpful the participants found these visual representations. See Additional file 2 for an example of these visual representations-radar diagrams.

\section{Qualitative responses}

For the purpose of tool design (refinements and additional characteristics), the current study design collected a wide range of responses from participants that will increase the tool's useability for practice facilitators. The specific suggestions include the following:

- An electronic tool that summarises and produces the visual representation

- Each stakeholder group needs own rating box/column

- Give the response options - 2 to +2 labels such as 'strongly agree', etc.

- Clarify questions to only address one concept at a time

Table 5 Summary of how helpful participants found the tool for planning, monitoring, and evaluation

\begin{tabular}{|c|c|c|c|c|c|c|}
\hline \multirow[t]{2}{*}{ Responses } & \multicolumn{2}{|c|}{ Planning } & \multicolumn{2}{|c|}{ Monitoring } & \multicolumn{2}{|c|}{ Evaluating } \\
\hline & $N$ & $\%$ & $N$ & $\%$ & $N$ & $\%$ \\
\hline Very helpful & 3 & 30 & 1 & 10 & 2 & 20 \\
\hline Helpful & 4 & 40 & 4 & 40 & 3 & 30 \\
\hline Neither helpful or unhelpful & 1 & 10 & 3 & 30 & 4 & 40 \\
\hline Unhelpful & 2 & 20 & 2 & 20 & 1 & 10 \\
\hline Very unhelpful & 0 & 0 & 0 & 0 & 0 & 0 \\
\hline Total & 10 & 100 & 10 & 100 & 10 & 100 \\
\hline
\end{tabular}

Table 6 Summary of how helpful participants found the visual representations

\begin{tabular}{lll}
\hline Visual representations & Number & Percentage \\
\hline Very helpful & 2 & 20 \\
Helpful & 3 & 30 \\
Neither helpful nor unhelpful & 4 & 40 \\
Unhelpful & 1 & 10 \\
Total & 10 & 100 \\
\hline
\end{tabular}

- Comparing against authors' scores was helpful

- Overlap between items-have facilitators narrow it down for what is meaningful

- Concerns that the tool is too subjective and user dependent to evaluate outcomes

- Simplify concepts and language

- Guidance on i-PARIHS domains/constructs prior to using the tool would be helpful

\section{Discussion}

Practice facilitators are responsible for facilitating the implementation of clinical innovations into practice. Therefore, it is important for them to successfully implement, then also evaluate and demonstrate this success. Implementation frameworks enable this, but the existence of these frameworks alone does not provide sufficient support or guidance for practice facilitators. Therefore, the current study introduced practice facilitators to an implementation framework, i-PARIHS, and engaged them in a workshop to try out and use a practical tool based on the i-PARIHS Facilitation Checklist that they can use in their everyday practice.

Overall, the attendees at the conference were highly engaged and enthusiastic about the i-PARIHS framework and the refinement and development of practical tools and resources. Many of these attendees highlighted their limited understanding and use of implementation frameworks and emphasised how they found the iPARIHS framework intuitively, very helpful. The survey results illustrate this enthusiasm and provide us with direction on the next steps needed for refinement and further development of the tool.

Specifically, we had three objectives. The first related to how practice facilitators found the workshop format and its components, as a way of being introduced to the i-PARIHS Facilitation Checklist. Participants had an overall positive experience with the workshop and found the introduction to implementation frameworks and the i-PARIHS framework valuable. This demonstrates that utilising conferences and interactive workshops are effective ways to educate practice facilitators on implementation science and introduce them to practical tools. Overall, this study supports the use of workshops as a way of introducing and spreading later iterations of the tool. 
Our second objective focused on whether practice facilitators found the i-PARIHS Facilitation Checklist tool useful and in what ways. Half of the participants reported they would use the tool in their everyday practice which supports the rationale for further development and refinement of practical i-PARIHS tools. The positive response to the tool suggests that the Facilitation Checklist [5] is useful in operationalising the i-PARIHS framework and adapting it into a useable tool was a useful starting point. Specifically, participants saw the tool as most helpful in planning their facilitation and implementation efforts, followed by monitoring these efforts and then evaluating their overall success. This demonstrates how practice facilitators find support in planning most crucial, and therefore important that we focus efforts on developing a tool that can provide this support.

The final objective related to tool characteristics that practice facilitators find most useful. This helps us decide what refinements to this first version of a tool are necessary. Participants appreciated the ability of the tool to enable a visual representation (radar diagram) of the iPARIHS dimensions, making it easy to determine which dimensions required the most facilitation. Through the qualitative feedback, participants encouraged the development of an electronic version of the tool which produces this visual representation, which supports its utility. Overall, the level of detail in which participants provided feedback on tool characteristics demonstrates their engagement and enthusiasm around its development.

As previous research identified, the original PARIHS framework received criticism for being difficult to operationalise [9] and the current study provides evidence that there remains demand and support for the development of i-PARIHS tools and resources to assist in using the iPARIHS framework in practice. Taking what we have learned from meeting our three objectives, our planned next steps for i-PARIHS tool development are to (i) continue to utilize the workshop format for sharing and refining tools, (ii) allocate a considerable component of our development efforts to tools that assist practice facilitators with planning, (iii) focus on modes of tool provision that keep user-friendliness in mind (e.g. through making electronic versions available), and (iv) identify what the key questions are from the i-PARIHS facilitation checklist and translate the checklist from academic language into more practical and user-friendly language.

\section{Limitations}

This study has several limitations. First, we had access to a limited number of participants for recruitment due to attendance at the workshop being completely optional and that the workshop was conducted at a conference that had concurrent sessions running. However, we felt it important to utilise this opportunity where a large number of practice facilitators were physically located at the one conference which resulted in greater success of recruitment compared with other means. Second, survey responses were not as rich as qualitative interviews, leaving some questions unanswered and detailed feedback limited. However, the time-efficiency associated with survey feedback that could be conducted at the time of the workshop outweighed the limitations. Finally, iPARIHS is an implementation framework that can be, and is used, across multiple settings (primary care, acute care, community care, etc.). Therefore, more work is required to actively involve and seek feedback from facilitators who work across different settings, not just practice facilitators working in primary care, as we move forward with our larger Mi-PARIHS program of work.

\section{Conclusions}

Despite a small sample size, this study was able to accomplish what we set out to do. Before embarking on a large program of work called the Mi-PARIHS Project where we will develop i-PARIHS tools and resources, we utilised this conference workshop opportunity, to gain some insight from those who are likely to use these tools and determine the need for their development, and what these users would like from i-PARIHS tools. This study has provided us with the reassurance that these tools will be used and will provide practical support for successful implementation.

Finally, this study serves as an example for other tool development endeavours. We have demonstrated how to effectively engage a convenient sample of end-users for feedback to direct and refine tool development, ensuring their feedback is utilised prior to a testing phase and is incorporated in the development stage. This study also demonstrates an effective way to disseminate and educate those who work in the field around the various theories and frameworks that support and inform their work. Overall, our approach could be tailored to other tool development endeavours' needs and the lessons learned through our results in this study (e.g. in terms of user preference) are likely to be applicable to those other development endeavours as well.

\section{Supplementary information}

Supplementary information accompanies this paper at https://doi.org/10. 1186/s43058-020-00046-0.

Additional file 1:. Itemised instrument.

Additional file 2:. Visual representations_radar diagrams.

Additional file 3:. Paper-based survey. 
NAPCRG: North American Primary Care Research Group; PARIHS: Promoting Action on Research Implementation in Health Services

\section{Acknowledgements}

This work is based on a conference presentation at the 2018 International Conference on Practice Facilitation, 10-11 December, Tampa, Florida.

\section{Authors' contributions}

SCH led the writing of the manuscript. SCH, BK, and ALK were key conceivers and designers of the study protocol. SCH and BK prepared and conducted the workshop and carried out the analysis of data. BK and ALK provided critical revisions to the manuscript's intellectual content, including but not limited to (i) contextualization of the content to the field's knowledge regarding implementation frameworks, practice facilitation, and co-design and (ii) structure of the results shared, which provide content area-specific findings of our work. All authors read and approved the final manuscript.

\section{Authors' information}

- SCH is a post-doctoral research fellow at Flinders University exploring Knowledge Translation and Implementation Science in clinical nursing practice and health care. She is also a visiting research fellow in the School of Psychology at the University of Adelaide. Her research interests include knowledge translation, evidence-based practice, nursing, men and masculinities in care work, fathering, and qualitative and discursive research. - BK is an investigator at the US Department of Veterans Affairs (VA) Center for Healthcare Organization and Implementation Research and an Instructor of Psychiatry at Harvard Medical School. As an implementation scientist for VA's Quality Enhancement Research Initiative, her research interests are in applying interdisciplinary methodologies toward studying the quality and implementation of health services.

- ALK is the Founding Director of the Caring Futures Institute, Flinders University and the inaugural Vice President and Executive Dean of the College of Nursing and Health Sciences at Flinders University, South Australia. Prior to this appointment she was Dean and Head of School at Adelaide Nursing School at the University of Adelaide. Her knowledge translation work is one of the most highly cited around the world.

\section{Funding}

This work is supported by author SCH's Knowledge Translation Post-Doctoral Research Fellowship and author BK's Center for Healthcare Organization and Implementation Research, neither of which played a role in the design of the study and collection, analysis, and interpretation of data and in writing the manuscript. The findings and conclusions in this article are those of the authors and do not represent the views of their affiliated institutions.

\section{Availability of data and materials}

All quantitative data collected or analysed during this study are included in this published article. Raw qualitative data are not publicly available due to them containing information that could compromise participants' privacy; derived data supporting the findings of this study are available from the corresponding author upon request.

\section{Ethics approval and consent to participate}

This study was approved by the Flinders University Social and Behavioural Research Ethics Committee (project number: 8223), and all participants provided informed consent.

\section{Consent for publication}

Not applicable

\section{Competing interests}

The authors declare that they have no competing interests.

\section{Author details}

'College of Nursing and Health Sciences, Flinders University, Sturt Road, Bedford Park, Adelaide, South Australia 5042, Australia. ${ }^{2}$ Caring Futures Institute, Flinders University, Sturt Road, Bedford Park, Adelaide, South Australia 5042, Australia. ${ }^{3}$ Center for Healthcare Organization and Implementation Research, US Veterans Health Administration, 150 South Huntington Avenue, Boston, MA 02130, USA. ${ }^{4}$ Department of Psychiatry, Harvard Medical School, 25 Shattuck Street, Boston, MA 02115, USA.
Received: 12 November 2019 Accepted: 5 June 2020

Published online: 18 June 2020

\section{References}

1. Blevins D, Farmer MS, Edlund C, Sullivan G, Kirchner JE. Collaborative research between clinicians and researchers: a multiple case study of implementation. Implement Sci. 2010;5(1):76.

2. Baskerville NB, Liddy C, Hogg W. Systematic review and meta-analysis of practice facilitation within primary care settings. The Annals of Family Medicine. 2012;10(1):63-74.

3. Mold JW, Fox C, Wisniewski A, Lipman PD, Krauss MR, Harris DR, Aspy C, Cohen RA, Elward K, Frame P, Yawn BP. Implementing asthma guidelines using practice facilitation and local learning collaboratives: a randomized controlled trial. The Annals of Family Medicine. 2014;12(3):233-40.

4. Schwarz RM. The skilled facilitator: A comprehensive resource for consultants, facilitators, managers, trainers, and coaches. Wiley. 2002.

5. Harvey G, Kitson A. PARIHS revisited: from heuristic to integrated framework for the successful implementation of knowledge into practice. Implement Sci. 2016;11(1):33.

6. Harvey G, Kitson A. Implementing evidence-based practice in healthcare: a facilitation guide. Routledge; 2015b.

7. Davis MM, Nagykaldi Z, Lipman PD, Haught J. ICPF Steering Committee. First international conference on practice facilitation: a success! The Annals of Family Medicine. 2018;16(3):274-5.

8. Bauer MS, Damschroder L, Hagedorn H, Smith J, Kilbourne AM. An introduction to implementation science for the non-specialist. BMC psychology. 2015;3(1):32.

9. Stetler $C B$, Damschroder $L$, Helfrich $C D$, Hagedorn HJ. A guide for applying a revised version of the PARIHS framework for implementation. Implement Sci. 2011;6(1):99.

10. Bauer MS, Miller CJ, Kim B, Lew R, Stolzmann K, Sullivan J, Riendeau R, Pitcock J, Williamson A, Connolly S, Elwy AR. Effectiveness of implementing a collaborative chronic care model for clinician teams on patient outcomes and health status in mental health: a randomized clinical trial. JAMA network open. 2019 Mar 1;2(3):e190230-.

11. Hsieh H-F, Shannon SE. Three approaches to qualitative content analysis. Qual Health Res. 2005613 Nov:15(9):1277-1288.

\section{Publisher's Note}

Springer Nature remains neutral with regard to jurisdictional claims in published maps and institutional affiliations.
Ready to submit your research? Choose BMC and benefit from:
- fast, convenient online submission
- thorough peer review by experienced researchers in your field
- rapid publication on acceptance
- support for research data, including large and complex data types
- gold Open Access which fosters wider collaboration and increased citations
- maximum visibility for your research: over $100 \mathrm{M}$ website views per year
At BMC, research is always in progress.
Learn more biomedcentral.com/submissions 